\title{
Molecular and Antigenic Characterization of the Leishmania (Viannia) panamensis Kinetoplastid Membrane Protein-11
}

\author{
José R Ramírez, Christof Berberich/+ , Andres Jaramillo, Carlos Alonso*, \\ Ivan D Vélez
}

\begin{abstract}
Programa de Estudio y Control de Enfermedades Tropicales, Sector de Inmunologia y Biología Molecular, Facultad de Medicina, Universidad de Antioquia, AA 1226, Medellín, Colombia *Centro de Biologia Molecular "Severo Ochoa", Universidad Autónoma de Madrid, Madrid, España

The kinetoplastid membrane protein 11 (KMP-11) has been recently described in Leishmania (Leishmania) donovani as a major component of the promastigote membrane. Two oligonucleotide primers were synthesized to PCR-amplify the entire coding region of New World Leishmania species. The Leishmania (Viannia) panamensis amplification product was cloned, sequenced and the putative amino acid sequence determined. A remarkably high degree of sequence homology was observed with the corresponding molecule of $\mathrm{L}$. (L) donovani and $\mathrm{L}$. (L) infantum $(97 \%$ and $96 \%$, respectively). Southern blot analysis showed that the KMP-11 locus is conformed by three copies of the gene. The $\mathrm{L} .(\mathrm{V})$ panamensis $O R F$ was subsequently cloned in a high expression vector and the recombinant protein was induced and purified from Escherichia coli cultures. Immunoblot analysis showed that 80\%, 77\% and 100\% sera from cutaneous, mucocutaneous and visceral leishmaniasis patients, respectively, recognized the recombinant KMP-11 protein. In a similar assay, $86 \%$ of asymptomatic Leishmania-infected individuals showed IgG antibodies against the rKMP-11. We propose that KMP-11 could be used as a serologic marker for infection and disease caused by Leishmania in America.
\end{abstract}

Key words: New World leishmaniasis - Leishmania (Viannia) panamensis - kinetoplastid membrane protein-11 antigenicity

The number of cases of American tegumentary leishmaniasis (ATL) has been estimated to be around 59,000 every year, and approximately 59 million people reside in areas where the ATL-causing Leishmania species are transmitted (Ashford et al. 1992). ATL caused by the species complex Leishmania (Viannia) braziliensis is endemic in large areas of Central and South America (Grimaldi et al. 1989). In Colombia, $L$. (V) panamensis is responsible for most of the ATL cases, including cutaneous leishmaniasis (CL), mucocutaneous leishmaniasis (MCL) and disseminated cutaneous leishmaniasis (DCL) (Corredor et al. 1990, Colombian Health Ministry 1994, Velez et al. 1994).

This work was supported, in part, by grants CIM9605 and CIM9750 from the Committee for the Development of Research (CODI) from the University of Antioquia and by the grant 1115-14436-95 from the Colombian Agency for the Development of Science and Technology (Colciencias). JR Ramírez is recipient of a $\mathrm{PhD}$ fellowship from Colciencias. A Jaramillo and ID Velez were recipients of investigator awards from Colciencias.

${ }^{+}$Corresponding author. Fax: + 57-4-511.4559

Received 29 October 1997

Accepted 15 January 1998
Control of leishmaniasis in the American continent is particularly complicated due to the fast evolving variety of Leishmania parasites and the complexity in their epidemiological patterns (Grimaldi \& Tesh 1993). Recent attempts to develop molecularly defined vaccines for leishmaniasis have focused on major parasite cell surface molecules, including the metalloproteinase GP63, the promastigote surface antigen complex (PSA) and the lipophosphoglycans (LPG) (McConville et al. 1987, Chang et al. 1990, Burns et al. 1991). For reasons such as its abundance on promastigotes and its important physiological role in parasitemacrophage interactions (Turco \& Descoteaux 1992), the latter was considered an attractive vaccine candidate. In early works, it has been demonstrated that immunization with LPG might confer immunoprotectivity in mice (Handman \& Mitchell 1985, McConville et al. 1987, Russell \& Alexander 1988) and that peripheral blood mononuclear cells from leishmaniasis patients were strongly stimulated to proliferate in response to LPG (McConville et al. 1987, Jaffe et al. 1990, Mendoca et al. 1991). However, it could be recently shown that T-cell stimulation associated with protective immunity elicited in mice, was due to a tightly associated small protein in the LPG fraction which was recently renamed as the kinetoplastid membrane pro- 
tein-11 (KMP-11) (Jardim et al. 1991, Stebeck et al. 1995). So far, KMP-11 has been cloned and partially characterized in $L .(L)$ donovani (Jardim et al. 1995a,b) and the $L$. $(L)$ infantum and Trypanosoma brucei rhodesiense counterparts have been recently reported (Stebeck et al. 1996, Berberich et al. 1997). To date, no homologous locus in any New World Leishmania species has been isolated and characterized. Here, we report the cloning, sequencing and bacterial expression of the recombinant $L .(V)$ panamensis KMP-11 protein. In addition, in a detailed study with sera from patients suffering from different forms of New World leishmaniasis we provide evidence that specific antibodies against KMP-11 are produced during natural course of infection.

\section{MATERIALS AND METHODS}

Parasites - Promastigotes of $L$. $(V)$ panamensis (MHOM/PA/71/LS94), L.(V) panamensis (MHOM /CO/87/UA140), L. (V) guyanensis (MHOM/BR/ 75/M4147), L. (L) major (MHOM/MA/81/ LEM265), L. (V) braziliensis (MHOM/CO/88/ $\mathrm{UA301}$ ), L. (V) colombiensis (IHAR/CO/85/ CL500), and L. (L) infantum (MHOM/FR/78/ LEM75), were grown at $26^{\circ} \mathrm{C}$ in Schneider's Drosophila Medium (Sigma, USA) supplemented with $15 \%$ heat-inactivated fetal bovine serum and antibiotics (streptomycin $0.01 \mathrm{mg} / \mathrm{ml}$, penicillin 10 $\mathrm{U} / \mathrm{ml}$ ). Promastigotes were grown to late logaritmic phase, harvested, washed three times with PBS and resuspended at $5 \times 10^{7} / \mathrm{ml}$.

Monoclonal and polyclonal antibodies - The L98 monoclonal antibody (mAb), originally raised against the $L$. $(L)$ donovani LPG molecule (Tolson et al. 1989), but further described to recognize a carboxy-terminal epitope on the tightly associated KMP-11 molecule (Jardim et al. 1991, 1995a), was used to demonstrate the presence of the recombinant KMP-11. This mAb detects the $11 \mathrm{kDa}$ protein in all kinetoplastid parasites so far tested (Stebeck et al. 1995). A rabbit polyclonal serum recognizing the recombinant $L$. (L) infantum KMP11 (Berberich et al. 1997) was also used to detect the recombinant $L(V)$ panamensis KMP-11 protein.

Study subjects - Twenty six patients with parasitological diagnosis of leishmaniasis were studied. The presence of Leishmania parasites in patients was determined by direct microscopic examination and culture methods. Indirect immunofluorescence microscopy using mAbs capable to distinguish between species-specific LPG epitopes (McMahon-Pratt \& David 1981) was applied in order to confirm the identity of Leishmania parasites. Twenty four patients had ATL caused by $L$. (V) panamensis or L. (V) braziliensis (15 CL and 9
MCL) and live in recognized endemic areas for leishmaniasis (departments of Antioquia, Tolima, Caldas and Cundinamarca, Colombia). Two patients had visceral leishmaniasis (VL) caused by L. $(L)$ chagasi/L. $(L)$ infantum and live in San Andres de Sotavento, an endemic region for VL at the Colombian Carribean Coast. ATL patients were treated as recommended by the WHO with Glucantime, $20 \mathrm{mg}$ of antimony $/ \mathrm{kg}$ daily intramuscularly (20 days for CL patients and 28 days for MCL patients) (WHO 1990). The two VL patients were treated with liposomal amphotericin B, $2 \mathrm{mg} /$ $\mathrm{kg}$ intravenously for 21 days. A second group consisted of seven asymptomatic Leishmania-infected individuals that live in endemic areas for leishmaniasis and show a positive Montenegro skin test (MST). These individuals were clinically evaluated to detect any scar or current leishmaniasis compatible lesion. As negative controls, nine healthy individuals which have never lived or visited endemic areas for leishmaniasis and have a negative MST were included. Serum samples were collected from these individuals in sterile vacutainer blood collection tubes (Becton, Dickinson and Company, Rutherford, NJ, USA) and frozen at $-20^{\circ} \mathrm{C}$ until use.

Polymerase chain reactions (PCR) - Genomic DNA was isolated as described previously (Medina-Acosta \& Cross 1993). To amplify the entire coding region of the KMP-11 protein from the genomic DNA, the following oligonucleotide primers were used: forward, (CB1): 5" ATGGCCACCACGTACGAGGAG 3" and reverse, (CB2): 5" GGACGGGTACTGCGCAGCC TT 3". The primer sequences were based on the published KMP-11 coding region from $L .(L)$ donovani (Jardim et al. 1995b). A "DNA thermal cycler 480" and "Amplitaq kit" (Perking-Elmer Cetus Instruments, New Jersey, USA) were used according to manufacturers instructions. Reactions mixtures included 25 pmol of each primer, $200 \mathrm{mM}$ of each dNTP and $1.5 \mathrm{mM} \mathrm{MgCl} 2$. The following PCR protocol was performed: (a) denaturation at $94^{\circ} \mathrm{C}$ for $1 \mathrm{~min}$; (b) annealing at $58^{\circ} \mathrm{C}$ for $1 \mathrm{~min}$; and (c) extension at $72^{\circ} \mathrm{C}$ for $1 \mathrm{~min} ; 30$ cycles.

Southern blot analysis - $3 \mathrm{mg}$ of $L .(V)$ panamensis promastigote genomic DNA were digested with restriction enzymes (Sal I, Nco I, Xho I, EcoR I, Hind III and BamH I), separated on a $0.8 \%$ agarose gel, transferred to a nylon membrane (Hybond-N, Amersham Int., UK) and subsequently hybridized with an $\left[a-{ }^{32} \mathrm{P}\right] \mathrm{dATP}$ random-primedlabelled probe consisting of the $273 \mathrm{bp} \mathrm{PCR} \mathrm{frag-}$ ment of the KMP-11 coding region. Hybridization was done in $6 \mathrm{X} \mathrm{SSC}$ (1X SSC is $0.15 \mathrm{M} \mathrm{NaCl}, 15$ $\mathrm{mM}$ Sodium-Citrate, $\mathrm{pH}$ 7.0), 0.1\% SDS, $250 \mathrm{mg} /$ $\mathrm{ml}$ hering sperm DNA and 50\% formamide, at 
$42^{\circ} \mathrm{C}$ overnight. After hybridization, membranes were washed with (a) $2 \mathrm{X} \mathrm{SSC}, 0.1 \%$ SDS; (b) $1 \mathrm{X}$ SSC, $0.05 \%$ SDS and (c) $0.1 \mathrm{X}$ SSC, $0.02 \%$ SDS, each washing step for $30 \mathrm{~min}$ at $42^{\circ} \mathrm{C}$. Membranes were then exposed to an autoradiographic film for $96 \mathrm{hr}$.

Cloning and sequencing - The 273 bp PCR fragment was blunt-end cloned in the EcoR V site of pBluescript (Stratagene, La Jolla, CA) and eight different recombinant clones were selected for restriction mapping to detect full-length inserts. Two of these clones were subsequently selected (clones pBx-KMP-4 and pBx-KMP-5) for DNA sequencing on double-stranded DNA by the dideoxy chain termination method (Sanger et al. 1977), using both comercial $\mathrm{T} 7$ and $\mathrm{T} 3$ primers or the $\mathrm{CB} 1 / \mathrm{CB} 2$ primer pair and the "sequenase kit" (US Biochemical Corp., Cleveland, OH, USA). The analysis of DNA sequence was done using the University of Wisconsin Genetics Computer Group Program (Devereux et al. 1984).

Subcloning, expression and purification of the recombinant protein - The $\mathrm{pBx}-\mathrm{KMP}-5$ insert was subcloned directionally into the BamH I/Hind III site of the expression plasmid pQE30 (Quiagen GMBH, Hilden, Germany). Induction of expression (2 mM IPTG) and protein purification was performed following the instructions of the manufacturer. Purification of proteins was performed under denaturing conditions in $8 \mathrm{M}$ Urea and passage of the bacterial extract through a Ni-NTA affinity column. Purified protein was subsequently dialysed against $1 \mathrm{X}$ PBS. The expression and purification process of the recombinant protein was monitored by SDS-PAGE and coomassie blue staining.

Western blotting - The purified recombinant protein was separated by $15 \%$ SDS-PAGE and electrotransferred to nitrocellulose membranes (Schleicher \& Schöll Inc., Keene, NH, USA) using the MiniTrans blot system (BioRad Labs, Hercules, CA, USA). Protein transfer was monitored by Ponceau-S (Sigma Co., St Louis, USA) staining before blocking the filters in 5\% blotto in PBS (Blocking solution) at $4^{\circ} \mathrm{C}$ overnight. The filters were then incubated with monoclonal (1:500), polyclonal $(1: 2000)$ or human $(1: 100)$ serum diluted in blocking solution with $0.5 \%$ tween-20 for $2 \mathrm{hr}$. To detect specific antibodies in human sera, the membranes were incubated successively with biotin-labelled anti-human IgG (Sigma Co., St Louis, USA) for $1 \mathrm{hr}$, streptavidin (BioRad Labs, Hercules, CA, USA) for $1 \mathrm{hr}$, and alkaline phosphatase-labelled biotin (BioRad Labs, Hercules, USA) for $1 \mathrm{hr}$. The immune reactions were visualized by incubating the membranes in bromochloroindolylphosphate-nitro blue tetrazolium
(BCIP-NBT) for $30 \mathrm{~min}$. For monoclonal and polyclonal sera, a peroxidase-labelled anti-mouse IgG and a alkaline phosphatase-labelled anti-rabbit IgG were used. Colour development was done with diaminobenzidine (DAB) and BCIP-NBT respectively.

\section{RESULTS}

Locus characterization and sequence of open reading frame - By using the $\mathrm{CB} 1 / \mathrm{CB} 2$ primer pair, which sequence was based on the $L$. $(L)$ donovani KMP-11 open reading frame (Jardim et al. 1995b), we amplified a 273-bp fragment in all species tested (Fig. 1) including L. (V) panamensis, L. (V) braziliensis and $L$. (V) guyanensis, the most important species causing CL and MCL in Colombia (Corredor et al. 1990, Colombian Health Ministry 1994). These results confirm, at the genomic level, the high degree of conservation for this gene between Old and New World Leishmania species as it was expected in view of the immunological crossreactivity of the protein in various kinetoplastid species when using the L98 and L157 mAbs originally raised against the LPG (Tolson et al. 1994).

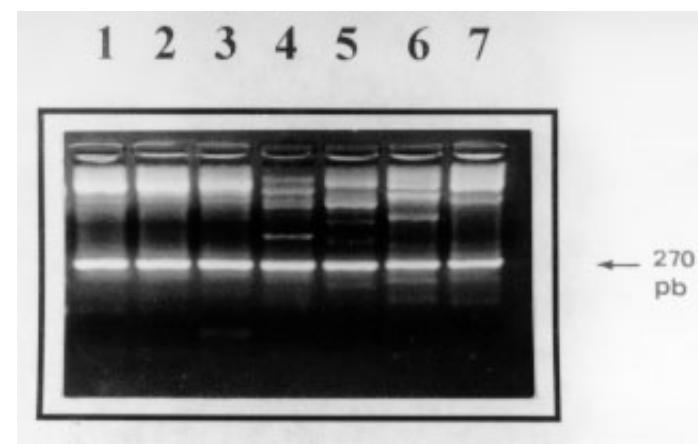

Fig. 1: PCR amplification of the KMP-11 gene in differents species of Leishmania using the CB1/CB2 primers. 1: $L$. (V) panamensis (LS94), 2: L. (V) panamensis (UA140), 3: L. (V) braziliensis (UA301), 4: L. (V) guyanensis (M4147), 5: L. (V) colombiensis (CL500), 6: L. (L) major (LEM265), 7: L. (L) infantum (LEM75). The amplification product of about $273 \mathrm{bp}$ is indicated.

The KMP-11 gene copy number of $L .(V)$ panamensis was determined by southern blot analysis using a variety of restriction endonucleases, including Sal I, which contains a cleavage site within the KMP-11 ORF, and others for which cleavage sites were not found within the gene (Nco I, Xho I, EcoR I, Hind III and BamH I). As shown in Fig. 2A, the Sal I restriction digests produced three DNA fragments that hybridized with the 273 bp KMP-11 PCR product used as probe, while the Xho I, EcoR I, Hind III and BamH I digests only revealed a single hybridizing DNA fragment. Three 
labelled bands were equally observed in the Nco I digested DNA representing fragments of 1.9, 3.0 and $7.6 \mathrm{~Kb}$, all different in size compared with the Sal I fragments of $1.4,1.8$ and $2.2 \mathrm{~Kb}$, thus indicating Nco I cleavage sites in intergenic regions. These results suggest that the KMP-11 locus consists of three gene copies separated by variable intergenic regions, although the presence in multiple genomic sites cannot be excluded. However, in view of the described genomic arrangement of the $L .(L)$ donovani and $L .(L)$ infantum KMP-11 genes (Jardim et al. 1995b, Berberich et al. 1997), the $L$. (V) panamensis counterparts should be probably organized in a similiar way as suggested in Fig. 2B. Differences in the genomic organization of the KMP-11 locus in Leishmania are only found in the lengh of the intergenic regions between each gene copy with variations from one species to another. In contrast to this Leishmania-specific genomic arrangement of the KMP-11 locus, the T. $b$. rhodesiense KMP-11 like gene is transcribed from a single copy gene (Stebeck et al. 1996).

A

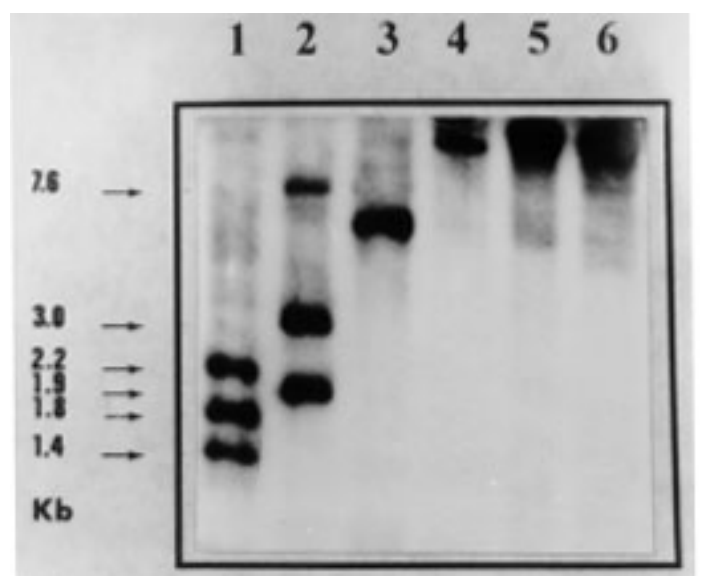

B

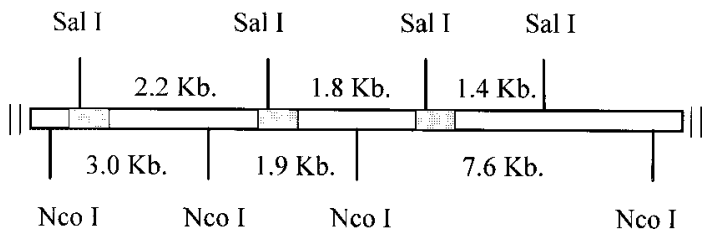

Fig. 2: genomic organization of the Leishmania (V) panamensis KMP-11 gene locus. A: southern blot analysis of restriction enzyme digested genomic DNA using as probe a $273 \mathrm{bp}$-fragment of the KMP-11 coding region. Restriction enzymes: 1 : Sal I, 2: Nco I, 3: Xho I, 4: EcoR I, 5: Hind III and 6: BamH I. The sizes of the labelled DNA fragments are indicated. B: schematic representation of the possible genomic organization of the KMP-11 locus. Shaded boxes indicate the position of the coding regions of the KMP-11 locus. The restriction sites and length of DNA fragments are indicated.
The nucleotide sequence of the PCR-amplified coding region of KMP-11 L. (V) panamensis gene is shown in Fig. 3 and is compared with the published $L .(L)$ infantum and $L$. $(L)$ donovani sequences (Jardim et al. 1995b, Berberich et al. 1997). A remarkably high level of sequence homology was observed between the aligned sequences $(97.1 \%, 96.3 \%$ and $82 \%$ for $L .(L)$ infantum, $L .(L)$ donovani and $T . b$. rhodesiense, respectively). The analysis of the deduced amino acid sequence predicts a putative $L$. $(V)$ panamensis KMP-11 protein with a molecular mass of $11,087 \mathrm{Da}$ and a calculated PI of 6.1. Only three amino acid changes were observed with respect to the sequences of the $L$. (L) infantum and $L$. $(L)$ donovani KMP-11 protein. The amino acid subtitutions were conservative in polarity.

Subcloning and bacteria expression - The 273 bp PCR fragment of the $L$. (V) panamensis KMP11 coding region was introduced in frame into the polylinker of the bacterial expression vector $\mathrm{pQE} 30$ (Quiagen, Hilden, Germany). Induction of recombinant protein expression by IPTG resulted in highlevel expression of the His-tagged KMP-11 with a molar mass of $14 \mathrm{kDa}$, slightly higher than the native protein due to the additional 6-His-tag and polylinker sequences. Purification of the recombinant protein was performed by affinity chromatography on Ni-NTA resin and the fractions were analyzed by SDS/PAGE. The results are shown in Fig. 4A. Subsequently, the purified protein was transferred to nitrocellulose membranes for western blot analysis. As shown in Fig. 4B, the rKMP-11 protein was strongly recognized by sera from VL patients known to contain high levels of anti-Leishmania antibodies (Sacks et al. 1993). A strong cross-reactivity was observed with a rabbit polyclonal anti-serum originally raised against the L. (L) infantum rKMP-11 protein (Berberich et al. 1997). In the same way, the $L 98 \mathrm{mAb}$ recognized specifically the $14 \mathrm{kDa}$ recombinant protein, confirming the identity of the rKMP-11 protein.

We also observed a second minor immunoreactive band of $28 \mathrm{kDa}$ in some western blots when using the polyclonal rabbit serum or sera from some leishmaniasis patients (Fig. 4B). That this band represents a dimeric form of the rKMP-11 due to its tendency to aggregate in vitro (Jardim et al. 1995, Berberich et al. 1997) was subsequently confirmed by using an antibody that specifically recognizes the His-tail in His-tagged proteins (data not shown). The lack of immunoreactivity of the $28 \mathrm{KDa}$ dimeric form in an immunoblot hybridized with the $\mathrm{L} 98 \mathrm{mAb}$ could be explained by the different accessibility between the monomeric and dimeric form. 


\begin{abstract}
$\begin{array}{lllllllllllllllllll}\mathbf{M} & \mathbf{A} & \mathbf{T} & \mathbf{T} & \mathbf{Y} & \mathbf{E} & \mathbf{E} & \mathbf{F} & \mathbf{S} & \mathbf{A} & \mathbf{K} & \mathbf{L} & \mathbf{D} & \mathbf{R} & \mathbf{L} & \mathbf{D} & \mathbf{E} & \mathbf{1 7}\end{array}$ L.pan ATGGCCACCACGTACGAGGAGTTTTCGGCGAAGCTGGACCGCCTGGATGA $\mathbf{5 0}$

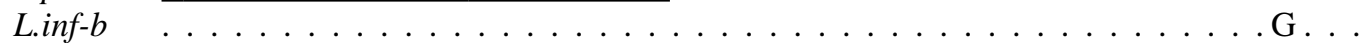

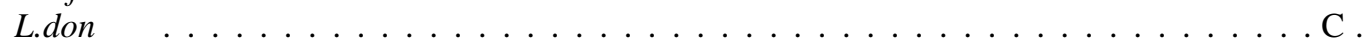

id $\begin{array}{lllllllllllllllll}\mathbf{E} & \mathbf{F} & \mathbf{N} & \mathbf{K} & \mathbf{K} & \mathbf{M} & \mathbf{Q} & \mathbf{E} & \mathbf{Q} & \mathbf{N} & \mathbf{A} & \mathbf{K} & \mathbf{F} & \mathbf{F} & \mathbf{A} & \mathbf{D} & \mathbf{3 3}\end{array}$ L.pan GGAGTTCAACAAGAAGATGCAGGAGCAGAACGCCAAGTTCTTTGCGGACA $\mathbf{1 0 0}$

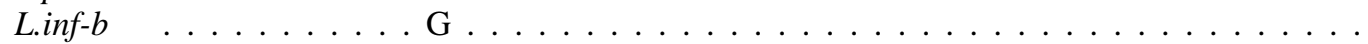
L.don
\end{abstract}

\title{
$d$
}

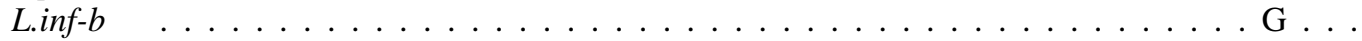

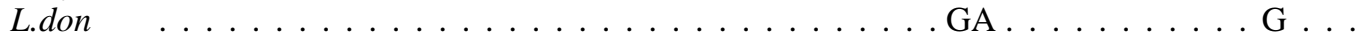

$\begin{array}{llllllllllllllllll}\mathbf{F} & \mathbf{E} & \mathbf{R} & \mathbf{M} & \mathbf{I} & \mathbf{K} & \mathbf{E} & \mathbf{H} & \mathbf{T} & \mathbf{E} & \mathbf{K} & \mathbf{F} & \mathbf{N} & \mathbf{K} & \mathbf{K} & \mathbf{M} & \mathbf{H} & \mathbf{6 7}\end{array}$ L.pan TTCGAGCGCATGATCAAGGAGCACACGGAGAAGTTCAACAAGAAGATGCA 200

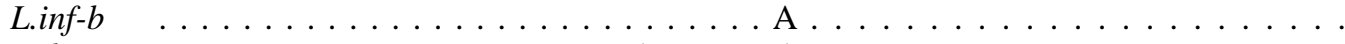
L.don $\ldots \ldots \ldots \ldots \ldots \ldots \ldots \ldots \ldots \ldots$

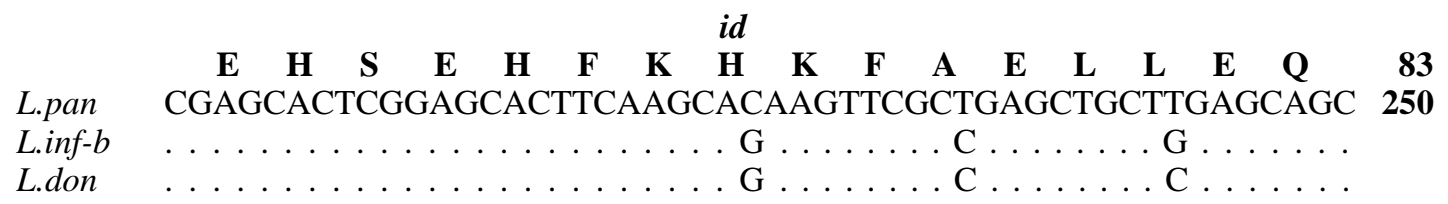

\section{$\begin{array}{llllllll}\mathbf{Q} & \mathbf{K} & \mathbf{A} & \mathbf{A} & \mathbf{Q} & \mathbf{Y} & \mathbf{P} & \mathbf{S}\end{array}$}

Fig. 3: sequence of the PCR-amplified Leishmania $(V)$ panamensis KMP-11 coding region. The sequence is compared with the coding region of Leishmania (L) infantum (gene B) (Berberich et al. 1997) and Leishmania (L) donovani KMP-11 gene (Jardim et al. 1995b). Dots indicate identical nucleotides and differences in the sequence are shown by the corresponding letters. Amino acid differences with regard to the $L$. $(L)$ infantum and $L .(L)$ donovani KMP-11 are indicated ( $i$ and $d$ respectively). Underlined nucleotides at the 5' and 3' ends represent the sequences of the CB1 and CB2 oligonucleotide primers used to PCR-amplify the coding region of $L$. (V) panamensis gene.

Antigenicity - The ability of the KMP-11 protein to induce proliferation of primed murine and human T-lymphocytes has been widely documented (Handman \& Mitchell 1985, McConville et al. 1987, Russell \& Alexander 1988, Jaffe et al. 1990, Jardim et al. 1991, Mendoca et al. 1991, Tolson et al. 1994). The B-cell antigenic potential has been also recently reported in dogs naturally infected with $L$. $(L)$ infantum (Berberich et al. 1997). To date, a systematic study about the presence of specific anti-KMP-11 anti-bodies in humans suffering from different forms of leishmaniasis has not been carried out. Therefore, in order to analyze the antigenicity of KMP-11 during New World Leishmania infections in humans, the re- combinant protein was assayed by western blots using sera obtained from CL, MCL and VL patients. In a similiar way, asymptomatic ("resistant") Leishmania-infected persons were tested.

The Table shows that the rKMP-11 protein was recognized by $80 \%(12 / 15)$ and $77 \%(6 / 7)$ of sera from CL and MCL patients, respectively, infected with $L$. (V) panamensis. A high degree of variability in the levels of anti-KMP-11 antibodies was observed in these groups of patients. However, sera from several CL and MCL patients showed a very strong reactivity, evidencing that the protein may act as a potent human B-cell immunogen. Both (100\%) of the human VL sera obtained from $L$. (L) chagasi-infected persons showed very strong 
A

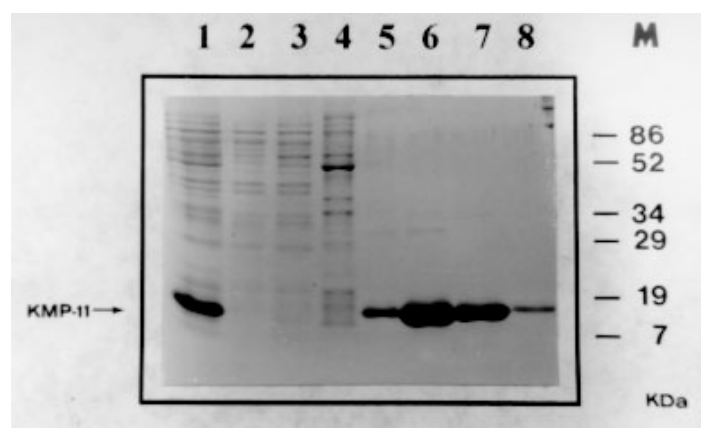

B

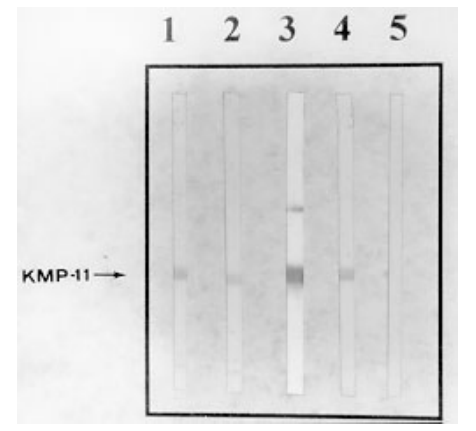

Fig. 4: Escherichia coli expression and western blot analysis of Leishmania. (V) panamensis KMP-11 protein. A: SDS-PAGE analysis of the expression and purification of the His-tagged recombinant KMP-11 protein. At each purification step, an aliquot was electrophoresed and the gel stained with coomasie blue. 1: supernatant phase of sonicated bacterial culture, 2: column flowthrough, 3 and 4 : washing fractions, 5, 6, 7 and 8: elution fractions, M: molecular weight marker. B: western blot of KMP-11 using serum of two VL patients $(1,2)$, rabbit polyclonal serum raised against the $L$. $(L)$ infantum rKMP-11 (3), L98 mAb (4) and negative control (no primary antibody was added) (5). The immunoreactive band corresponding to a $14 \mathrm{kDa}$ protein is indicated.

immunoreactivity against the heterologous KMP11 (Table) from $L$. (V) panamensis, consistent with the high levels of anti-Leishmania antibodies observed in these patients (Sacks et al. 1993). In addition, the immunological cross-reactivity between these VL sera and the $L .(V)$ panamensis protein confirms the high level of conservation of KMP11 between species causing either CL, MCL or VL.

The potent B-cell immunogenic role of KMP11 was also demonstrated when assaying sera from asymptomatic Leishmania-infected persons in western blots. A total of $86 \%$ (6/7) of these sera were seropositive for KMP-11, although, in general, the immunoreactivity was weak compared with the titers observed in most of the CL, MCL and VL sera. The low level of anti-Leishmania antibodies in these asymptomatic infections is consistent with the predominant Th1-like cellular immune reponse found in autohealing CL and patients

\section{TABLE}

Western blot reactivity of human sera against Leishmania (V) panamensis KMP-11. The immunoreactivity was semi-quantitatively evaluated and according to the intensity of the signals marked with one to four crosses. The minus sign means no reactivity. In the first group, 15 CL sera (CB 1-15), 9 MCL sera (W 1-9) and 2 VL sera (VL 2-3) were tested. In the second group 7 sera from asymptomatic MST positive individuals (R 1-7) and 9 sera from healthy MST negative individuals (CB 45-50, ESTBLC 1-3) were also tested

\begin{tabular}{|c|c|c|c|}
\hline \multicolumn{2}{|c|}{ Reactivity } & \multicolumn{2}{|c|}{ Reactivity } \\
\hline \multicolumn{4}{|c|}{ Group 1} \\
\hline \multicolumn{2}{|c|}{$\begin{array}{l}\text { Cutaneous } \\
\text { leishmaniasis }\end{array}$} & \multicolumn{2}{|c|}{$\begin{array}{l}\text { Mucocutaneous } \\
\text { leishmaniasis }\end{array}$} \\
\hline CB1 & +++ & W1 (3106) & + \\
\hline CB2 & + & W2 (1872) & + \\
\hline CB3 & ++ & W3 (2129) & ++ \\
\hline CB4 & + & W4 (1702) & - \\
\hline CB5 & + & W5 (3114) & - \\
\hline CB6 & ++ & W6 (2105) & + \\
\hline CB7 & - & W7 (2326) & +++ \\
\hline CB8 & + & W8 (2534) & + \\
\hline CB9 & - & W9 (1874) & +++ \\
\hline CB 10 & + & & \\
\hline CB11 & + & & \\
\hline CB12 & ++ & \multicolumn{2}{|c|}{ Visceral leishmaniasis } \\
\hline CB13 & + & & \\
\hline CB14 & ++ & VL2 & ++++ \\
\hline CB 15 & + & VL3 & ++++ \\
\hline \multicolumn{4}{|c|}{ Group 2} \\
\hline \multicolumn{2}{|c|}{$\begin{array}{l}\text { Asymptomatic } \\
\text { MST positive }\end{array}$} & \multicolumn{2}{|c|}{$\begin{array}{c}\text { Healthy } \\
\text { MST negative }\end{array}$} \\
\hline R1 & + & CB45 & - \\
\hline $\mathrm{R} 2$ & - & CB46 & - \\
\hline $\mathrm{R} 3$ & + & CB47 & - \\
\hline R4 & + & CB48 & - \\
\hline R5 & + & CB49 & - \\
\hline R6 & + & CB50 & - \\
\hline \multirow[t]{3}{*}{ R7 } & + & ESTBLC1 & - \\
\hline & & ESTBLC2 & - \\
\hline & & ESTBLC3 & - \\
\hline
\end{tabular}

without clinical symptoms of leishmaniasis (Carvalho et al. 1995). The negative control group (non-Leishmania infected persons) in contrast, did not show any reactivity against KMP-11 indicating lack of natural IgG antibodies against this protein.

\section{DISCUSSION}

Recent reports on the cloning and molecular characterization of the KMP-11 protein in the Old World Leishmania species $L .(L)$ donovani and $L$. $(L)$ infantum (Jardim et al. 1995b, Berberich et al. 1997) and in the Trypanosoma species T. $b$. rhodesiense (Stebeck et al. 1996) revealed similiar 
structural features of this kinetoplastid-specific protein, although differences in the genomic arrangement and in the pattern of distribution were found that may influence its function. The present study was carried out to analyze the sequence and gene locus organization of KMP-11 in the New World Leishmania species $L$. (V) panamensis, the prevalent etiological agent of cases of CL and MCL in Colombia (Corredor et al. 1990, Colombian Health Ministry 1994). Here, we provide evidence that the sequence of KMP-11 is highly conserved (> 95\% homolgy) in all Leishmania species so far analyzed as well as the genomic organization consisting of three gene copies, regardless of the epidemiological pattern and the clinical symptoms of leishmaniasis they cause. The $T$. $b$. rhodesiense counterpart of this protein, in contrast, shows $18 \%$ divergence with respect to Leishmania and the gene is transcribed from a single gene copy (Stebeck et al. 1996). Immunofluorescence studies also showed that the $L .(L)$ donovani (Stebeck et al. 1995), the $L$. (L) infantum (C Berberich, manuscript in preparation) and the T. $b$. rhodesiense (Stebeck et al. 1996) KMP-11 exhibit different fluorescence signals pointing to strict species-specific differences in the distribution of this protein.

The biological function of this protein is still far from being clear. Recently, it has been speculated that the monomethylarginine in the $L$. $(L)$ donovani KMP-11 sequence on position 45 might be involved in parasite-defense mechanisms (Jardim et al. 1995a) after partial degradation of the protein in the parasitophorus vacuole. This modified arginine residue is known as a competitive inhibitor of nitric oxide synthetase, a central enzyme involved in generation of the anti-Leishmania radical nitric oxide within activated murine macrophages (Liew et al. 1990). It was proposed that within the strongly hydrolytic conditions of the phagolysosome, the KMP-11 protein would be released from the parasite and degraded to amino acids by proteolytic enzymes. Subsequently the inhibitory monomethylarginine would contribute to the enzyme inhibition and parasite survival (Jardim et al. 1995a). However, in the $L$. (V) panamensis sequence as well in the $L$. $(L)$ infantum (Berberich et al. 1997) and T. b. rhodesiense (Stebeck et al. 1996) molecules, this arginine was substituted by a lysine and should therefore not account for such a speculative function. Since the consistent co-purification of a minor component together with the full-length $L$. $(L)$ donovani KMP11 indicates a specific proteolytic event and, since major post-translational modifications including Oglycosylation and $\mathrm{N}$-methylation of this protein seem to occur (Jardim et al. 1995a), we cannot, however, exclude that a monomethylarginine resi- due from another position might be responsible for such an effect. Both, the exact pattern of post-translational modifications and a potential inhibitory role of putative monomethylarginines in the KMP11 molecule remain to be determined. So far, what can be assumed is, that the high level of sequence conservation of KMP-11 together with the wide distribution throughout kinetoplastid parasites, suggest an essential role of this protein in the parasite biology. Protein modeling analysis and detailed CD-experiments (Jardim et al. 1995b, M Fuertes $\&$ C Berberich, manuscript in preparation) suggest that KMP-11 has a highly amphipatic character with an unusually high degree of alpha-helical structure sharing important thermodynamic features with the class of apolipoproteins (Ryan et al. 1993). It has been therefore hypothezised, that the KMP-11 protein could be involved in the stabilization of the parasite membrane. However, recent findings of a strict developmentally regulated and stage-specific expression together with a defined cellular localization mainly around the flagellum and flagellar pocket in $L .(L)$ infantum and T. $b$. rhodesiense (Stebeck et al. 1996, C Berberich, manuscript in preparation) suggests additional functions.

The detailed antigenicity analysis of the recombinant KMP-11 of $L$. (V) panamensis in human CL, $M C L$ and VL sera suggests that this protein acts as a potent B-cell immunogen during natural course of disease. Our results show that $82 \%$ (27/33) of Leishmania-infected humans (symptomatic or asymptomatic) contain detectable levels of antiKMP-11 IgG antibodies (Table). In general, a high degree of variability in the anti-KMP-11 antibody level was observed in CL and MCL sera and a stronger immunoreactivity is associated with symptomatic infections when compared with the asymptomatic form. Therefore, the high antigenicity of KMP-11 may point to a potential serological marker for diagnosis of infection and disease. However, in view of the immunological cross-reacitivity observed against the T. cruzi counterpart of KMP11 (Stebeck et al. 1995) it may be necessary to determine specific epitopes in KMP-11 capable to distinguish between leishmaniasis and Chagas disease. Future studies will also concentrate on the Tcell antigenicity in all forms of human and mouse leishmaniasis in order to evaluate KMP-11 as a possible vaccine candidate.

\section{REFERENCES}

Ashford R, Desjeux P, Deraadt P 1992. Estimation of population at risk of infection and number of cases of leishmaniasis. Parasitol Today 8: 104-105.

Berberich C, Requena JM, Alonso C 1997. Cloning of genes, expression and antigenicity analysis of the 
Leishmania infantum KMP-11 protein. Exp Parasitol 85: 105-108.

Burns J, Scott J, Carvalho E, Russo D, March C, Vanness K, Reed S 1991. Characterization of a membrane antigen of Leishmania amazonensis that stimulates human immune responses. J Immunol 146: 742-748.

Carvalho EM, Correia D, Bacellar O, Almeida R, Lessa $\mathrm{H}$, Rocha H 1995. Characterization of the immune response in subjets with self-healing cutaneous leishmaniasis. Am J Trop Med Hyg 53: 273-277.

Chang KP, Chaudhhuri G, Fong D 1990. Molecular determinants of Leishmania virulence. Ann Rev Microbiol 44: 499-529.

Colombian Health Ministry 1994. Leishmaniosis, plan nacional de control. Manual de normas tecnicoadministrativas, Trazo Ltda, Santafe de Bogotá, 106 pp.

Corredor A, Kreutzer R, Tesh R, Boshell J, Palau M, Caceres E, Duque S, Pelaez D, Rodriguez G, Nichols S, Hernandez C, Morales A, Young D, Ferro C 1990. Distribution and etiology of leishmaniasis in Colombia. Am J Trop Med Hyg 42: 206-214.

Devereux J, Haeberli O, Smithies O 1984. A comprehensive set of sequence analysis programs for the VAX. Nucleic Acids Res 12: 387-395.

Grimaldi G, Tesh R 1993. Leishmaniasis of the New World: current concepts and implications for the future research. Clin Microbiol Rev 6: 230-250.

Grimaldi G, Tesh R, McMahon-Pratt D 1989. A review of the geographic distribution and epidemiology of leishmaniasis in the New World. Am J Trop Med Hyg 41: 687-725.

Handman E, Mitchell GF 1985. Immunization with Leishmania receptor for macrophages protecs mice against cutaneous leishmaniasis. Proc Natl Acad Sci USA 82: 5910-5914.

Jaffe CL, Shor R, Tau H, Passwell J 1990. Parasite antigens recognized by patients with cutaneous leishmaniasis. Clin Exp Immunol 80: 77-82.

Jardim A, Funk V, Caprioli R, Olafson R 1995a. Isolation and structural characterization of the Leishmania donovani kinetoplastid membrane protein-11, a major immunoreactive membrane glycoprotein. Biochem J 305: 307-314.

Jardim A, Hanson S, Ullman B, McCubbin W, Kay C, Olafson R 1995b. Cloning and structure-function analysis of the Leishmania donovani kinetoplastid membrane protein-11. Biochem J 305: 315-320.

Jardim A, Tolson D, Turco S, Pearson T, Olafson R 1991. The Leishmania donovani lipophosphoglycan Tlymphocyte reactive component is a tightly associated protein complex. J Immunol 147: 3538-3544.

Liew FY, Li Y, Millot S 1990. Tumor necrosis factor-a synergizes with interferon-gin mediating killing of Leishmania major through the induction of nitric oxide. J Immunol 145: 4306-4310.

McConville MJ, Bacic A, Mitchell GF, Handmann E 1987. Lipophosphoglycan of Leishmania major that vaccinates against cutaneous leishmaniasis contains an alkylglycerophosphoinositol lipid anchor. Proc Natl Acad Sci USA 84: 8941-8945.

McMahon-Pratt D, David JR 1981. Monoclonal anti- bodies that distinguish between New World species of leishmania. Nature 291: 581-583.

Medina-Acosta E, Cross G 1993. Rapid isolation of DNA from trypanosomatid protozoa using a simple "miniprep" procedure. Mol Biochem Parasitol 59: 327-330.

Mendoca SCF, Russel DG, Coutinho SG 1991. Analysis of the human T-cell responsiveness to purified antigens of Leishmania lipophosphoglycan (LPG) and glycoprotein 63 (GP63). Clin Exp Immunol 83: 472-478.

Russell DG, Alexander J 1988. Effective immunization against cutaneous leishmaniasis with defined membrane antigens reconstituted into liposomes. $J$ Immunol 140: 1274-1279.

Ryan RO, Oikawa K, Kay CM 1993. Conformational, thermodynamic and stability properties of Manducta sexta apolipophorin III. J Biol Chem 268: 1525-1530.

Sacks D, Louis J, Wirth D 1993. Leishmaniasis, p237239. In KS Warren Immunology and Molecular Biology of Parasitic Infections, 3rd edition, Blackwell Scientific Publication, Boston.

Sanger F, Nicklen S, Coulson AR 1977. DNA sequencing with chain-terminating inhibitors. Proc Natl Acad Sci USA 74: 5463-5467.

Stebeck CE, Baron G, Beecroft R, Pearson T 1996. Molecular characterization of the kinetoplastid membrane protein-11 from African trypanosomes. Mol Biochem Parasitol 81: 81-88.

Stebeck CE, Beecroft R, Singh B, Jardim A, Olafson R, Tuckey C, Prenevost K, Pearson T 1995. Kinetoplastid Membrane Protein-11 (KMP-11) is differentially expressed during the life cycle of African trypanosomes and is found in a wide variety of kinetolastid protozoan parasites. Mol Biochem Parasitol 71: 1-13

Tolson DL, Jardim A, Schnur L, Stebeck C, Tuckey C, Beecroft R,Teh H, Olafson R, Pearson T 1994. The kinetoplastid membrane protein-11 of Leishmania donovani and African trypanosomes is a potent stimulator of T-lymphocyte proliferation. Infect Immun 62: 4893-4899.

Tolson DL, Turco SJ, Beecroft RP, Pearson TW 1989. The immunochemical structure and surface arrangement of Leishmania donovani lipophos-phoglycan determined by monoclonal antibodies. Mol Biochem Parasitol 35: 109-118.

Turco SJ, Descoteaux A 1992. The lipophosphoglycan of Leishmania parasites. Ann Rev Microbiol 46: 6594.

Velez ID, Agudelo S, Robledo S, Jaramillo L, Segura I, Soccol V, Restrepo S 1994. Diffuse cutaneous leishmaniasis with mucosal involvement in Colombia caused by an enzymatic variant of Leishmania panamensis. Trans R Soc Trop Med Hyg 88: 199-199.

Weigle KA, Dedavalos M, Heredia P, Molineros R, Saravia N, D’Alessandro A 1987. Diagnosis of cutaneous and mucocutaneous Leishmaniasis in Colombia: a comparison of seven methodos. Am J Trop Med Hyg 36: 489-496.

WHO - World Health Organization 1990. Expert commitee on control of the leishmaniasis. Tech Rep Ser: 793. 